Original Article

\title{
Effects of wearing rubber gloves on activities of the forearm and shoulder muscles during different dishwashing stages
}

\author{
Won-Gyu Yoo ${ }^{1)}$ \\ 1) Department of Physical Therapy, College of Biomedical Science and Engineering, Inje University: \\ 607 Obangdong, Gimhae, Gyeongsangnam-do 621-749, Republic of Korea
}

\begin{abstract}
Purpose] The present study examined the effects of wearing rubber gloves on the activities of the forearm and shoulder muscles during two dishwashing stages. [Subjects] This study included 10 young females. [Methods] The participants performed two dishwashing stages (washing and rinsing) with and without rubber gloves. The activities of the wrist flexor and upper trapezius muscles were measured using wireless electromyography. [Results] During the washing stage, the activities of the wrist flexor and upper trapezius muscles were significantly greater without gloves than with gloves when performing the same tasks. However, during the rinsing stage, the activities of these muscles did not differ significantly according to the use of gloves. [Conclusion] Dishwashers should wear gloves during the washing stage to prevent wrist and shoulder pain.

Key words: Dishwasher, Rubber gloves, Shoulder pain
\end{abstract}

(This article was submitted Mar. 13, 2015, and was accepted Apr. 16, 2015)

\section{INTRODUCTION}

Cleaning tasks such as washing cookware entail high workloads involving repetitive hand and wrist movements ${ }^{1)}$. Typically, many dishes are washed in a large, deep sink. During a busy work shift, dishwashers bend the trunk at an angle of $10-45^{\circ}$ from an upright position and maintain this fixed posture for long periods without a break ${ }^{2}$. Studies have evaluated the effects of load during dishwashing on the low back and legs ${ }^{3)}$. Although most studies have focused on low back pain in dishwashers, dishwashing also puts excessive load and tension on the forearm and shoulder muscles ${ }^{3,4)}$. A prolonged poor posture with a forward head position and protracted shoulders could contribute to neck and shoulder pain ${ }^{5)}$

The use of rubber gloves is a common practice in many industries, including meat packing, construction, and warehousing. Wearing gloves can alter the efficiency of tasks that require manual dexterity, although few studies have investigated this. Therefore, this study examined the effects of wearing rubber gloves on the activities of the forearm and shoulder muscles during two dishwashing stages.

Corresponding author. Won-gyu Yoo (E-mail: won7y@inje. ac.kr)

C2015 The Society of Physical Therapy Science. Published by IPEC Inc. This is an open-access article distributed under the terms of the Creative Commons Attribution Non-Commercial No Derivatives (by-ncnd) License $<$ http://creativecommons.org/licenses/by-nc-nd/3.0/>.

\section{SUBJECTS AND METHODS}

Ten female students (mean age, $33.1 \pm 2.2$ years; height, $158.9 \pm 3.0 \mathrm{~cm}$; weight, $57.2 \pm 5.7 \mathrm{~kg}$ ) participated in this study. All of the participants were right-hand dominant and typically spent approximately 1 hour per day washing dishes at home. The purpose and methods of the study were explained to the participants before their inclusion in the study, and they provided informed consent according to the principles of the Declaration of Helsinki.

The activities of the left wrist flexor and upper trapezius muscles were measured using a wireless electromyography (EMG) system (Delsys, Boston, MA, USA). The EMG data are expressed as a percentage of the maximum voluntary isometric contraction. The participants performed two dishwashing stages (washing and rinsing) with and without rubber gloves, washing five 20-cm-diameter dishes at their usual speed. The height of the dishwashing table was adjusted such that the top was level with the anterior superior iliac spines of the participants.

Statistical analysis was performed using SPSS software for Windows ver. 18.0 (SPSS, Chicago IL, USA) with the level of statistical significance set at $p<0.05$. A paired t-test was used to assess differences in the activities of the wrist flxor and upper trapezius muscles between conditions with and those without rubber gloves during the two dishwashing stages.

\section{RESULTS}

During the washing stage, the activities of the wrist flexor and upper trapezius muscles were significantly $(\mathrm{p}<0.05)$ greater without gloves than with gloves $(51.4 \pm 22.0 \%$ vs. 
$40.5 \pm 10.9 \%$ and $32.1 \pm 13.8 \%$ vs. $26.6 \pm 9.0 \%$, respectively). During the rinsing stage, the activities of the wrist flexor and upper trapezius muscles did not differ significantly ( $p$ $>0.05$ ) between conditions with and those without gloves $(37.4 \pm 19.1 \%$ vs. $35.5 \pm 17.9 \%$ and $20.1 \pm 13.8 \%$ vs. $22.6 \pm$ $12.0 \%$, respectively).

\section{DISCUSSION}

Research can help industries select the types of rubber gloves that enable the most efficient performance while providing suitable protection for a specific job. This study showed that during the dish washing stage, the activities of the wrist flexor and upper trapezius muscles were significantly higher without gloves than with gloves. In the present study, the activities of the wrist flexor and upper trapezius muscles during the rinsing stage did not differ significantly according to the use of glove. Recent reports have suggested that the use of rubber gloves increases the incidence of cumulative traumatic disorders and interferes with the wearer's ability to perform fine motor activities and object manipulations ${ }^{5,6)}$. Information gained from studies on gloves can help set guidelines for designing workplaces and equipment. A decrease in forearm muscle activation was shown to lead to a compensatory increase in shoulder movements ${ }^{5,6)}$. Shin and Yoo found that manual material handling or light manual precision work depended on exerting effective grip force ${ }^{7)}$. The grip force exerted was reduced by impaired recruitment of the extensor digitorum and flexor digitorum superficialis, resulting in an increased incidence of wrist or shoulder pain $^{8)}$. Known risk factors for upper-extremity muscle injury include repetitive and continuous detailed work ${ }^{9}$.

Typically, most dishwashers wear poorly fitting gloves that allow their hands to move inside the gloves, impairing fine-motor performance ${ }^{10)}$. Wearing the wrong gloves can affect manual dexterity, which is key to performing many tasks efficiently ${ }^{10)}$. However, working bare handed or wearing well-fitting rubber gloves was shown to result in effective forearm muscle activation, which decreased inefficient shoulder movements ${ }^{8}$. Wearing well fitting rubber gloves can improve safety and comfort in the workplace, helping manual workers to perform their tasks both rapidly and efficiently ${ }^{8)}$. In conclusion, dishwashers should wear gloves during the washing stage to prevent wrist and shoulder pain.

\section{REFERENCES}

1) Sakai K, Watanabe A, Onishi N, et al.: Features and workload in hospital food service operation. J Sci Labour, 1993, 69: 240-252.

2) Iwakiri K, Yamauchi S, Yasukouchi A: Effects of a standing aid on loads on low back and legs during dishwashing. Ind Health, 2002, 40: 198-206. [Medline] [CrossRef]

3) Iwakiri K, Sotoyama M, Mori I, et al.: Does leaning posture on the kitchen counter alleviate workload on the low back and legs during dishwashing? Ind Health, 2007, 45: 535-545. [Medline] [CrossRef]

4) Iwakiri K, Sotoyama M, Mori I, et al.: Shape and thickness of cushion in a standing aid to support a forward bending posture: effects on posture, muscle activities and subjective discomfort. Ind Health, 2004, 42: 15-23. [Medline] [CrossRef]

5) Rempel DM, Harrison RJ, Barnhart S: Work-related cumulative trauma disorders of the upper extremity. JAMA, 1992, 267: 838-842. [Medline] [CrossRef]

6) Jensen C, Finsen L, Hansen K, et al.: Upper trapezius muscle activity patterns during repetitive manual material handling and work with with a computer mouse. J Electromyogr Kinesiol, 1999, 9: 317-325. [Medline] [CrossRef]

7) Shin SJ, Yoo WG: Effects of below-knee assembly work at different reach distances on upper-extremity muscle activity. J Phys Ther Sci, 2014, 26: 1277-1278. [Medline] [CrossRef]

8) Bensel CK: The effects of various thicknesses of chemical protective gloves on manual dexterity. Ergonomics, 1993, 36: 687-696. [Medline] [CrossRef]

9) Yoo And IG, Yoo WG: Changes in the cervical FRR, shoulder muscle pain and position after continuous detailed assembly work. Work, 2014, 49: 735-739. [Medline]

10) Drabek $T$, Boucek $C D$, Buffington $C W$ : Wearing the wrong size latex surgical gloves impairs manual dexterity. J Occup Environ Hyg, 2010, 7: 152-155. [Medline] [CrossRef] 